\title{
Reachability Analysis of Planar Multi-linear Systems ${ }^{\star}$
}

\author{
Oded Maler ${ }^{1}$ and Amir Pnueli ${ }^{2}$ \\ 1 VERIMAG, B.P. 53x, 38041 Grenoble, France \\ 2 The Weizmann Institute of Science, Rehovot 76100, Israel.
}

\begin{abstract}
In this paper we study the reachability analysis of a simple class of hybrid systems, namely multi-linear systems. Such systems consist of a partition of the Euclidean space into a finite set of polyhedral sets (regions). Within each region the dynamics is defined by a constant vector field, hence the discrete transitions occur only on the boundaries between the regions where the trajectories change their direction. Such a system can be described by a finite set of guarded commands where the guards are conjunctions of linear inequalities and the commands are differential equations with constant right hand sides, corresponding to the vector fields.

Our goal is to verify, based on the description of the system, various reachability questions between regions of the plane. In particular we show that in planar deterministic systems, the question whether there exists a trajectory connecting a state in a source region to a state in a target region is decidable.
\end{abstract}

\section{Introduction}

Hybrid systems (HS) are systems that combine intercommunicating discrete and continuous components. Most embedded systems belong to this class since they operate and interact with a continuous environment, and are expected to provide real-time responses to continuously varying situations.

The introduction of HS models is motivated by a real practical concern: with the decrease in the size and price of computing elements, more and more computers (discrete transition systems) are embedded within real-world control loops such as in avionics, process control, robotics and consumer products - to mention a few application areas. The analysis and prediction of the combined behavior of these embedded systems require formal tools that cut across existing disciplinary boundaries: the real-world is usually modeled by control engineers as a continuous dynamical system while computer scientists investigate the dynamics of discrete systems.

The ultimate goal of the theory of HS will be to build models of such embedded systems, models which include the dynamics of an external environment and

* This research was supported in part by the France-Israel project for cooperation in Computer Science, and by the European Community ESPRIT Basic Research Action Projects REACT (6021) 
the interface between the controller and the environment. Within these models, based upon a description of a discrete controller (such as a program or a digital circuit) and upon its timing characteristics, it will be possible to prove that the behavior of the controlled environment satisfies certain properties. Even if we cannot realistically hope for fully algorithmic analysis techniques, any progress along this line of research will enhance the quality of current design methodologies, and will provide system developers with models and with software tools that will result in a more efficient, systematic and reliable development process.

\subsection{Models for Hybrid Systems}

Hybrid systems generalize both discrete transition systems and continuous dynamical systems. A HS consists of two types of state-variables: discrete variables whose values change via discrete transitions, and continuous variables whose values change according to some continuous dynamical law in any time interval of positive length. These two types of variables interact with each other in the following ways:

1. Some property satisfied by continuous variables (e.g., a variable crosses a threshold) enables or disables a discrete transition.

2. A change in a discrete variable may change the dynamical rule to which some continuous variables are subject.

Several models for hybrid systems have been proposed recently (see [5], [8], [6], [7], [2], [4]) together with some negative and positive results concerning the decidability of verification problems. The positive results usually involved the special case of timed graphs/automata [1], [3], whose introduction was motivated by real-time systems. Timed graphs can be viewed as hybrid systems where the only continuous variables are timers, all increasing at the same constant rate, and possibly being reset when discrete transitions occur. Tests on values of those timers serve as "guards" for performing the discrete transitions - usually those tests are conjunctions of simple linear inequalities in one variable.

Similar to [4] (Integration Graphs), we are looking for decidable subclasses of Constant Slope Hybrid Systems (CSHS). The strategy taken in [4] is based on placing restrictions on the guards of transitions that may occur within loops. Here we take an alternative approach and allow arbitrary boolean combinations of linear inequalities as transition guards, but place the following restrictions:

- The system is deterministic.

- Transitions do not modify any of the continuous variables. Thus, there are no resets or other assignment statements.

- The control component of a state is uniquely determined by the values of the continuous variables.

It follows that trajectories of such systems are continuous, but not smooth. Systems obeying such requirement are closer to continuous dynamical systems and are more amenable to topological and geometrical analysis. 
Due to the fact that the discrete (control) component of the state is fully determined by the values of the continuous variables and that all changes are continuous, we prefer to present the system without explicit reference to discrete states. Instead, the system is viewed as a set of regions (corresponding to discrete states in other presentations) with boundaries separating them. Each region is associated with a vector field which identifies the rates at which the various variables change. Reaching a boundary and crossing into another region is equivalent to taking a transition to another discrete state in which the continuous evolution rule is different.

\subsection{An Example: Cat and Mouse II}

In order to motivate the reader we will present a toy problem which is analyzable using the techniques developed in this paper.

The Problem Consider cat and a mouse located in a one-dimensional space. Their respective positions are denoted by the state-variables $x$ and $y$ and we assume that initially $x>y$. The line is divided into two parts, illuminated (left) and dark (right). The behavior of each of them consists of running either left or right in certain velocities based on their relative locations. Usually the cat tries to minimize its distance from the cat while the mouse tries to increase the distance (they do it in different velocities according to the distance). There are two exceptions: when the cat is on the dark side and too far away, the mouse mistakenly thinks that it is a female mouse and tries to minimize the distance. On the other hand when the mouse is in the illuminated side, the cat mistakenly thinks that it is Mighty Mouse and runs away (see figure 1).

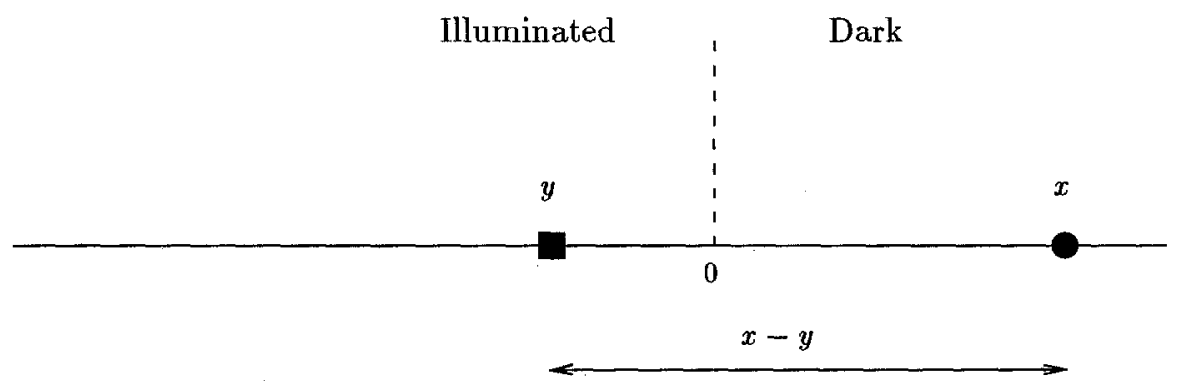

Fig. 1. The cat $x$ and the mouse $y$.

The precise behavioral rules of the cat and the mouse are depicted in table 1 . All the parameters are positive and the entries in the tables denote velocities. 


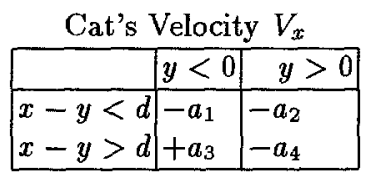

Mouse's Velocity $V_{y}$
\begin{tabular}{|c|c|c|}
\hline & $x<0$ & $x>0$ \\
\hline$x-y<d$ & $-b_{1}$ & $+b_{2}$ \\
$x-y>d$ & $-b_{3}$ & $-b_{4}$ \\
\hline
\end{tabular}

Table 1. The behavioral rules of the cat and the mouse.

Knowing all the parameters of the system we would like to answer questions such as: Given that the cat starts at some position in the interval $\left[x_{0}, x_{1}\right]$, and that the mouse starts at some position in $\left[y_{0}, y_{1}\right]$, will they ever meet $(x=y)$ ? Is it possible that the distance between them will become larger then some $d^{*}$ ? Will the mouse ever reach some point $x^{*}$ ?

The Solution If we look at the mouse and the cat positions as the coordinates of our system, we obtain a planar (2-dimensional) multi-linear system (see exact definitions below). Each point in the $x, y$ plane represents the joint positions of the cat and the mouse. Their corresponding rules of behavior induce a partition of the plane into regions such that within every region the system evolves with a constant slope of the form $c=\left(a_{i}, b_{j}\right)$ as depicted in figure 2 .

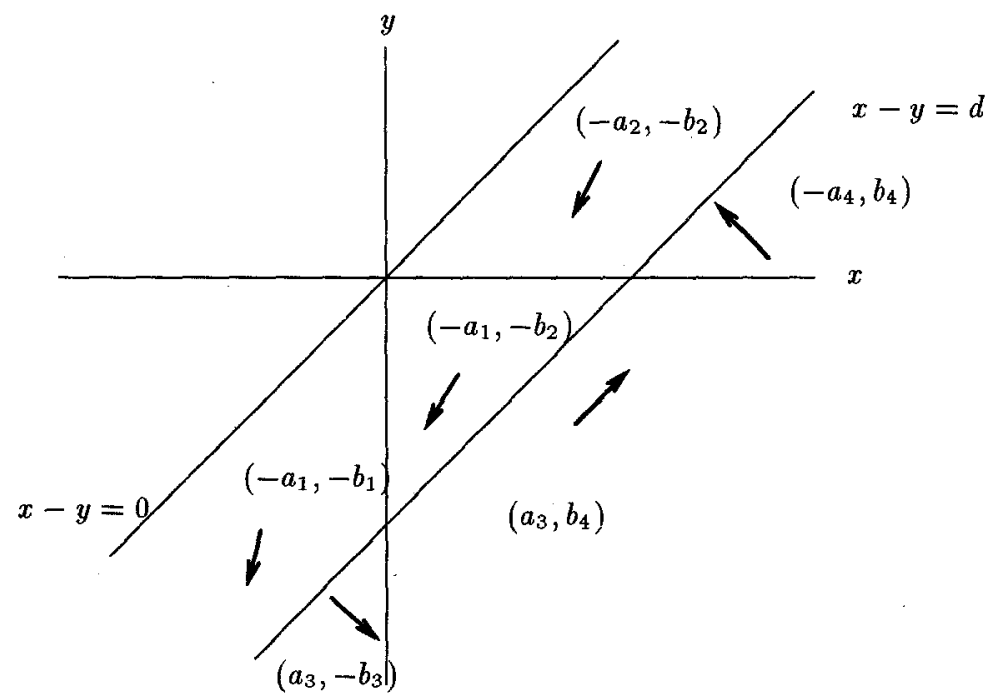

Fig. 2. The cat $x$ and the mouse $y$ viewed as a planar multi-linear system.

In this paper we show how for every system of this type, reachability questions between polyhedral subsets of the state-space can be effectively answered. 


\section{Preliminaries}

Polyhedral Partitions, Systems and Trajectories Throughout this paper, we deal with $X=\mathbb{R}^{d}$ considered as a $d$-dimensional Euclidean space with a metric $\rho$. Points (or vectors) in $X$ are denoted by $\mathbf{x}$ or by a according to the context. The expression $\mathbf{a} \cdot \mathbf{x}$ denotes an inner product. The interior $\operatorname{int}(C)$ of $C \subseteq X$ is the set of points $\mathbf{x} \in C$ such that there exist a neighborhood $N_{\epsilon}(\mathbf{x}) \subseteq X$ satisfying $N_{\epsilon}(\mathbf{x}) \subseteq C$. The boundary of $C$ is then $b d(C)=c l(C)-i n t(C)$, where $c l(C)$ denotes the closure of $C$. For every vector $\mathbf{x}=\left(x_{1}, x_{2}\right) \in X^{2}$ we define its right rotation as the vector $\hat{\mathbf{x}}=\left(x_{2},-x_{1}\right)$. Clearly $\mathbf{x} \cdot \hat{\mathbf{x}}=0$ and $\mathbf{x} \cdot \mathbf{y}=\hat{\mathbf{x}} \cdot \hat{\mathbf{y}}$.

An open linear set (half-space) in $X$ is the set of all points $\mathbf{x} \in X$ satisfying $\mathbf{a} \cdot \mathbf{x}+b<0$ (we assume that all the coefficients are rational). A polyhedral set is an intersection of finitely many linear sets. Every polyhedral set is convex. We will assume without loss of generality that all polyhedral sets are given in a non-redundant form, that is, by omitting any half-space from the intersection we obtain a different set. We will also assume that they are all $d$-dimensional. This paper concentrates on the two-dimensional case where linear sets are called halfplanes and polyhedral sets are called polygonal sets, but some of the definitions and the analysis techniques are more general.

Definition 1 Polyhedral partition. A finite polyhedral partition of $X$ is a family $\mathcal{P}=\left\{P_{1}, \ldots, P_{k}\right\}$ of polyhedral sets such that $\bigcup_{i=1}^{k} \operatorname{cl}\left(P_{i}\right)=X$ and for every $P_{i}, P_{j} \in \mathcal{P}, P_{i} \cap P_{j}=\emptyset$.

We will denote by $b d(\mathcal{P})$ the set of all points in $X$ which are in $b d(P)$ for some $P \in \mathcal{P}$ and by $E(\mathcal{P})$ the set of edges of $\mathcal{P}$, namely non-empty subsets of $X$ of the form $e=\operatorname{int}\left(\operatorname{cl}\left(P_{i}\right) \cap \operatorname{cl}\left(P_{j}\right)\right)$ for some $P_{i}, P_{j} \in \mathcal{P}$. Similarly the set of vertices of $\mathcal{P}, V(\mathcal{P})$ consists of points $\mathbf{x} \in X$ such that $\{\mathbf{x}\}=\operatorname{cl}\left(e_{i}\right) \cap \operatorname{cl}\left(e_{j}\right)$ for some $e_{i}, e_{j} \in E(\mathcal{P})$. We call the elements of $B(\mathcal{P})=E(\mathcal{P}) \cup V(\mathcal{P})$ border elements. One can easily see that $X$ is decomposed into a disjoint union $\mathcal{P} \cup E(\mathcal{P}) \cup V(\mathcal{P})$. For example, the polyhedral partition in figure 3 has 5 regions, 3 vertices and 7 edges.

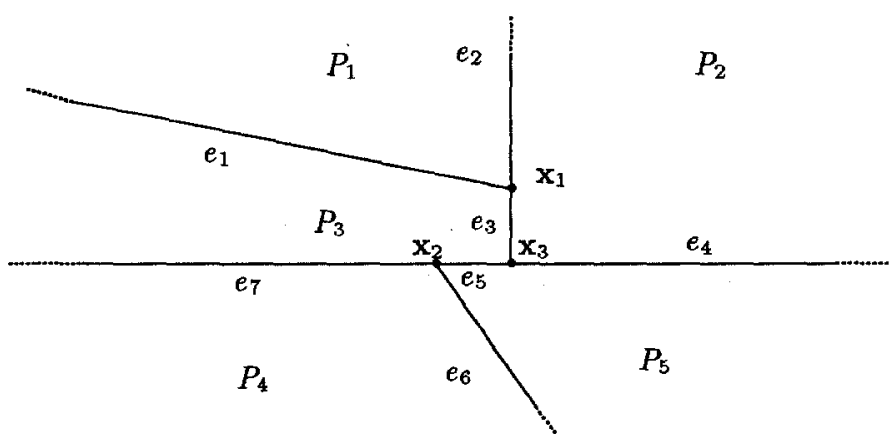

Fig. 3. A polyhedral partition of the plane. 
Suppose $e$ is an edge such that $e \subseteq b d(P) \cap b d\left(P^{\prime}\right)$. Let $P=\left\{\mathbf{x}: \bigwedge_{i \in I} \mathbf{a}_{i}\right.$. $\left.\mathbf{x}+b_{i}<0\right\}$ and $P^{\prime}=\left\{\mathbf{x}: \bigwedge_{i^{\prime} \in I^{\prime}} \mathbf{a}_{i^{\prime}} \cdot \mathbf{x}+b_{i^{\prime}}<0\right\}$. Then for $e$ to be non-empty there must be some $j \in I, k \in I^{\prime}$, such that $\mathbf{a}_{j}=-\mathbf{a}_{k}$ and $b_{j}=-b_{k}$ and every $\mathbf{x} \in e$ satisfies $\mathbf{a}_{j} \cdot \mathbf{x}+b_{j}=0$. We call $\mathbf{a}_{j}$ and $\mathbf{a}_{k}$ the characteristic vectors of $e$ relative to $P$ and $P^{\prime}$. One can see that they are two opposite normals to $e$.

Definition 2 Multi-linear systems. A multi-linear dynamical hybrid system on $X$ is $\mathcal{H}=(\mathcal{P}, \varphi, \psi)$ where $\mathcal{P}$ is a polyhedral partition of $X, \varphi: \mathcal{P} \rightarrow X$ is a function which assigns to each region a vector (slope) in $X$, and a function $\psi: B(\mathcal{P}) \rightarrow \mathcal{P}$ satisfying $e \subseteq \operatorname{cl}(\psi(e))$ for every $e \in B(\mathcal{P})$.

The intuitive meaning (to be formalized by the definition of a step) is that $d \mathbf{x} / d t=\varphi\left(P_{i}\right)$ for every $\mathbf{x} \in P_{i}$, and that every boundary element is associated with one of the regions for which it is a boundary - this way we impose determinism on the boundaries. We denote $\varphi\left(P_{i}\right)$ by $\mathbf{c}_{i}$.

Definition 3 Step. A step of a multi-linear system is a pair of boundary points $\left(\mathbf{x}, \mathbf{x}^{\prime}\right)$ such that $\mathbf{x} \in e, \mathbf{x}^{\prime} \in e^{\prime}, e, e^{\prime} \subseteq b d(P)$ for some region $P, \psi(e)=P$, $\varphi(P)=\mathbf{c}$ and there exists some $t>0$ such that $\mathbf{x}^{\prime}=\mathbf{x}+\mathbf{c t}$. The set of reachable states during such a step is $R_{\mathbf{X}, \mathbf{x}^{\prime}}=\left\{\mathbf{x}+\mathbf{c} t^{\prime}: 0 \leq t^{\prime} \leq t\right\}$.

Definition 4 Trajectory. A trajectory of a system starting at point $\mathrm{x} \in X$ is a sequence $\xi=\mathbf{x}_{0}, \mathbf{x}_{1}, \mathbf{x}_{2}, \ldots$ such that, for every $i \geq 0,\left(\mathbf{x}_{i}, \mathbf{x}_{i+1}\right)$ is a step. We will say that $\mathbf{x}_{i+1}$ is a successor of $\mathbf{x}_{i}$.

Ttrajectories can be infinite (they cross boundaries indefinitely) or finite (they enter a "terminal" unbounded region and stay there). We deal with deterministic systems in the sense that to every $\mathbf{x} \in X$ there is exactly one corresponding trajectory. The reachable states of a trajectory are defined naturally. Although we have chosen to define trajectories via their discrete "samplings", we will also use this term to denote the underlying continuous curve.

Orientation of Boundary Points Consider a region $P$ and one of its edges $e$ with a characteristic vector a relative to $P$, and let $\mathbf{c}=\varphi(P)$. Then, by simple calculation one can see that $e$ is an entry to $P$ if $\mathbf{a} \cdot \mathbf{c}>0, e$ is an exit from $P$ if $\mathbf{a} \cdot \mathbf{c}<0$, and $e$ is tangent to $P$ if $\mathbf{a} \cdot \mathbf{c}=0$. For the simplicity of presentation we assume that the system is not degenerate, i.e., no vector field is parallel to an edge of its region $(\mathbf{a} \cdot \mathbf{c} \neq 0)$.

For every vertex $\mathrm{x} \in b d(P)$ there are exactly two edges $e, e^{\prime} \subseteq b d(P)$ such that $\mathbf{x}=\operatorname{cl}(e) \cap c l\left(e^{\prime}\right)$. Then one can see that $\mathbf{x}$ is an entry point to $P$ if both $e$ and $e^{\prime}$ are entry entry edges. Symmetrically it is an exit point if both $e$ and $e^{\prime}$ are exit edges. Otherwise, i.e., when one of $\left\{e, e^{\prime}\right\}$ is an entry edge and the other is an exit edge we say that $\mathbf{x}$ is neutral w.r.t. $P$. This way we have completed the classification of all boundary points according to their orientation relative to the region (see figure 4). We will denote the set of all entry points of $P$ by $\operatorname{In}(P)$ and set of all exit points by $\operatorname{Out}(P)$. 


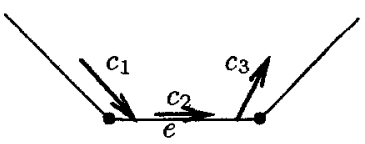

(a)

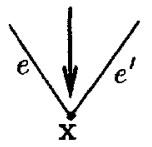

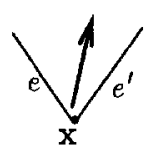

(b)

Fig.4. The possible orientations between a region and its borders: (a) $e$ is an exit, tangent entry to $P$ according to whether $\varphi(P)$ is $\mathbf{c}_{1}, \mathbf{c}_{2}$ or $\mathbf{c}_{3}$ respectively. (b) $\mathbf{x}$ is exit, entry, or neutral w.r.t. $P$.

\section{Properties of Planar Deterministic Systems}

In this section we prove some fundamental properties of planar systems which apply to an even more general class than the multi-linear systems considered in this paper. The only necessary condition is that any edge can be traversed in at most one direction.

Ordering the Boundaries Consider a region $P$ whose boundary is partitioned into $\operatorname{In}(P), \operatorname{Out}(P)$ and a pair of neutral points $\left\{\mathbf{z}_{1}, \mathbf{z}_{2}\right\}$. The separator of $P$ is the line $l$ connecting $\mathbf{z}_{1}$ and $\mathbf{z}_{2}$. The mappping $\theta: X \rightarrow \mathbb{R}$, defined as $\theta(\mathbf{x})=$ $\mathbf{x} \cdot\left(\mathbf{z}_{1}-\mathbf{z}_{2}\right)$, assigns to every $\mathbf{x}$ a value proportional to the lenghth of the projection of the vector $\mathbf{x}$ on $l$. Then one can easily see that the relation $\preceq$, defined as $\mathbf{x}_{1} \preceq \mathbf{x}_{2}$ if $\theta\left(\mathbf{x}_{1}\right) \leq \theta\left(\mathbf{x}_{2}\right)$, is a dense linear order on $\operatorname{In}(P)$ and $\operatorname{Out}(P)$ (see figure 5).

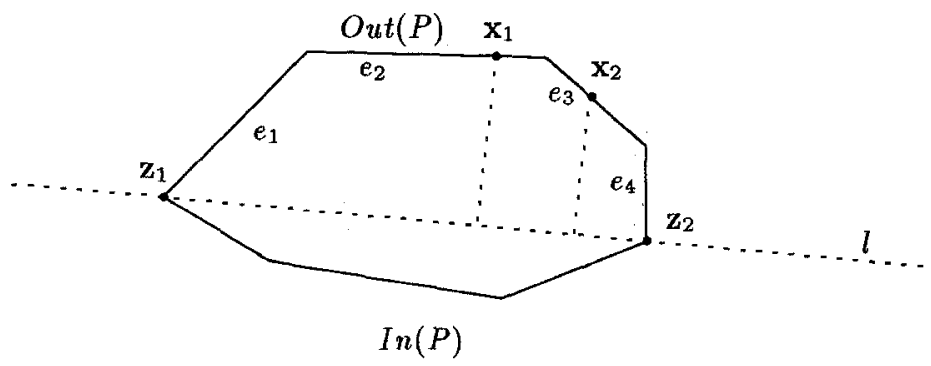

Fig. 5. Ordering on the boundary: $\mathbf{x}_{1} \preceq \mathbf{x}_{2} ; e_{1} \prec e_{2} \prec e_{3} \prec e_{4}$.

The fact that $\operatorname{In}(P)$ and $\operatorname{Out}(P)$ are ordered allows us to speak of boundary intervals of the form $\left[\mathbf{x}_{1}, \mathbf{x}_{2}\right]$ denoting all the points $\mathbf{x} \in \operatorname{In}(P)$ (or $\operatorname{Out}(P)$ ) satisfying $\mathbf{x}_{1} \preceq \mathbf{x} \preceq \mathbf{x}_{2}$. We use $\prec$ to denote the strict variant of $\preceq$ and will say that $e_{1} \prec e_{2}$ if $\mathbf{x}_{1} \prec \mathbf{x}_{2}$ for every $\mathbf{x}_{1} \in e_{1}, \mathbf{x}_{2} \in e_{2}$. For example, in figure 5 we have $e_{1} \prec e_{2} \prec e_{3} \prec e_{4}$.

Remark: In this definition we only considered the case of bounded regions those must have 2 neutral points. For unbounded regions ("cones") one can easily see that there are two cases. Either they don't have any neutral points 
and cannot be traversed more than once by a trajectory - and thus are of no interest to us for the purpose of the forthcoming result. In the case the region $P$ has one neutral point $z$ we can take the separator to be any line $l$ satisfying $\ln b d(P)=\{\mathbf{z}\}$.

Claim 1 (Fundamental property of planar systems) Let $\xi$ be any trajectory that intersects $\operatorname{In}(P)$ (or Out $(P)$ ) in three consecutive points, $\mathrm{x}_{1}, \mathrm{x}_{2}$ and $\mathbf{x}_{3}$. Then, $\mathbf{x}_{1} \preceq \mathbf{x}_{2}$ implies $\mathbf{x}_{2} \preceq \mathbf{x}_{3}$ and $\mathbf{x}_{1} \succeq \mathbf{x}_{2}$ implies $\mathbf{x}_{2} \succeq \mathbf{x}_{3}$.

Proof: If $\mathbf{x}_{1}=\mathbf{x}_{2}$ then due to determinism $\mathbf{x}_{2}=\mathbf{x}_{3}$. So we assume $\mathbf{x}_{1} \prec \mathbf{x}_{2}$ and show that $\mathbf{x}_{3} \prec \mathbf{x}_{2}$ implies an intersection of $\xi$ with itself. Suppose, without loss of generality, that the trajectory from $\mathbf{x}_{1}$ to $\mathbf{x}_{2}$ circumvents $P$ from the left (that is, its last intersection with the separator line $l$ before reaching $\mathbf{x}_{2}$ is some $\mathbf{y} \prec \mathbf{x}_{1}$ ) (see figure 6). Consider now the closed set $S$ bounded by the closed curve consisting of the trajectory from $\mathbf{x}_{1}$ to $\mathbf{x}_{2}$ and the boundary interval $\left[\mathbf{x}_{1}, \mathbf{x}_{2}\right]$. In order that there will be a trajectory form $\mathbf{x}_{2}$ to $\mathbf{x}_{3}$ there must be two points, $x_{2}^{\prime}$ "above" $x_{2}$ and $\mathbf{x}_{3}^{\prime}$ "below" $x_{3}$ such that there is a trajectory $\mathbf{x}_{2} \rightarrow \mathbf{x}_{2}^{\prime} \rightarrow \mathbf{x}_{3}^{\prime} \rightarrow \mathbf{x}_{3}$ and in particular we can choose $\mathbf{x}_{2}^{\prime}$ outside $S$ and $\mathbf{x}_{3}^{\prime}$ inside $S$ (if we cannot then $\mathbf{x}_{2}$ and $\mathbf{x}_{3}$ coincide). Consequently, according to Jordan theorem, the trajectory $\mathbf{x}_{2}^{\prime} \rightarrow \mathbf{x}_{3}^{\prime}$ must intersect the boundary of $S$. Since it cannot do it on $\left[\mathbf{x}_{1}, \mathbf{x}_{2}\right], \xi$ must intersect itself and this contradicts determinism unless $\mathbf{x}_{2}=\mathbf{x}_{3}$. This is true independent of whether the trajectory from $\mathbf{x}_{2}$ to $\mathbf{x}_{3}$ circumvents $e$ from the left (figure 6-a), or from the right (figure 6-b).

This topological property has many consequences concerning the set of possible trajectories. It implies that the sequence of consecutive intersection points of a trajectory with $\operatorname{In}(P)$ or $O u t(P)$ is monotone w.r.t. $\preceq$. In fact, the relation between $\mathbf{x}_{1}, \mathbf{x}_{2}$ and $\mathbf{y}$ determines two classes of possible "quasi-cycles": if $\mathbf{x}_{2}$ is between $\mathbf{x}_{1}$ and $\mathbf{y}$ we have a "contracting spiral" (fig 7 -a) while if $\mathbf{x}_{1}$ is between $\mathbf{x}_{2}$ and $\mathbf{y}$ we have an "expanding spiral" (fig 7-b). Once a trajectory has performed a contracting spiral, it will never reach any point "outside" the spiral (i.e., outside the closed curve formed by the trajectory $\mathbf{x}_{1} \rightarrow \mathbf{y} \rightarrow \mathbf{x}_{2}$ and the boundary interval $\left.\left[\mathbf{x}_{1}, \mathbf{x}_{2}\right]\right)$ and, symmetrically, after performing an expanding spiral, a trajectory will never reach a point "inside" the spiral.

Signatures The qualitative behavior of a trajectory $\xi: \mathbf{x}_{1}, \mathbf{x}_{2}, \ldots$ is captured by its signature, which is the sequence of border elements (edges and vertices), $\sigma(\xi)=e_{1}, e_{2}, \ldots$ such that for every $i, \mathbf{x}_{i} \in e_{i}$. The sequences $\rho(\xi)=P_{1}, P_{2}, \ldots$ is the corresponding region signature of $\xi$ satisfying the obvious relation $e_{i} \subseteq$ $\operatorname{Out}\left(P_{i}\right) \cap \operatorname{In}\left(P_{i+1}\right)$.

A sequence $w=w_{1}, \ldots, w_{n}$ over some finite alphabet is called a cycle if $w_{1}=w_{n}$ and $\left\{w_{i}, \ldots, w_{n-1}\right\}$ are mutually disjoint. A sub-trajectory $\xi_{[i . . j]}=$ $\mathbf{x}_{i}, \mathbf{x}_{i+1}, \ldots, \mathbf{x}_{j}$ forms a region cycle if its region signature, $P_{i}, \ldots, P_{j}$ is a cycle. It forms an edge cycle if its edge signature $e_{i}, \ldots, e_{j}$ is a cycle. Note that if $\xi_{[i . . j]}$ is an edge cycle it is also a region cycle but not vice versa).

An edge $e$ is said to be abandoned by a trajectory after position $i$, if $e_{i}=e$ and for some $j, k, i \leq j<k, \xi_{[j, . . k]}$ forms a region cycle and $e \notin\left\{e_{i+1}, \ldots, e_{k}\right\}$. 


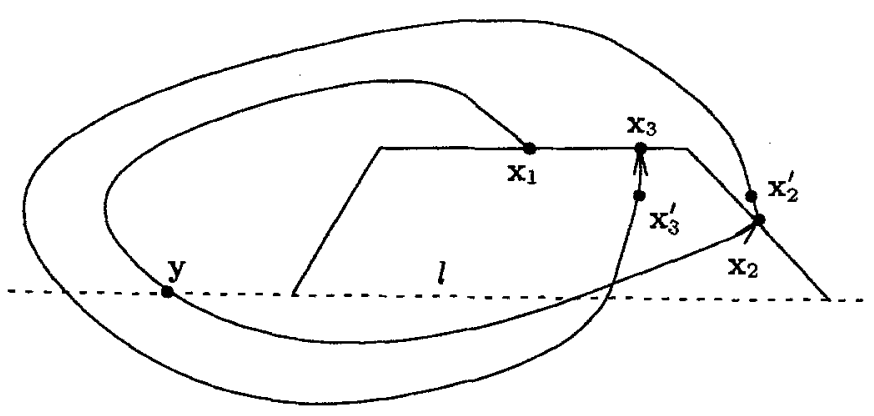

(a)

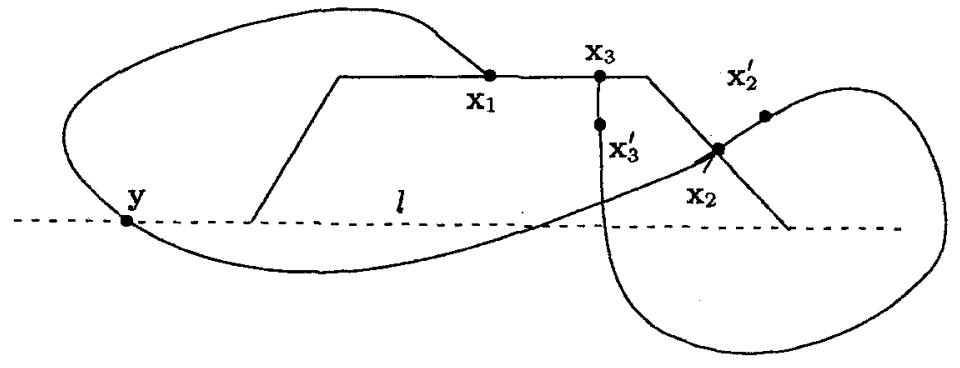

(b)

Fig. 6. Proof of the fundamental property: (a) Circumvention from the left. (b) Circumvention from the right.

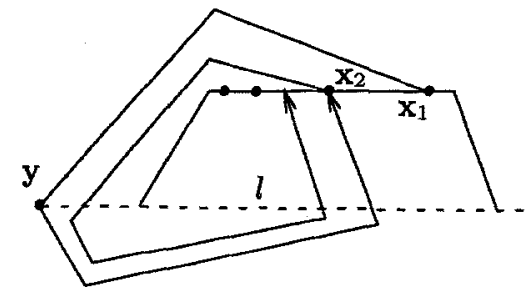

(a)

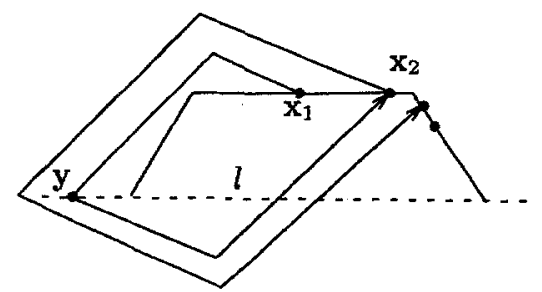

(b)

Fig. 7. (a) A contracting spiral. (b) An expanding spiral.

Claim 2 (Abandonment is Irreversible) An edge $e$ abandoned after position $i$ will not appear in the signature at any position $m>i$.

Proof: Let $\xi_{[j . . k]}$ be the trajectory that forms the relevant region cycle. We assume without loss of generality that $\mathbf{x}_{j} \prec \mathbf{x}_{k}$ and that the cycle circumvents $P_{j}$ from the left (expanding spiral). Consider the case of $i=j$. Then, another intersection of $\xi$ with $e_{i}$ must happen at some $\mathbf{x}_{m} \prec \mathbf{x}_{k}$ and this will violate the monotonicity property (see figure 8-a). For the case $i<j, e_{i}$ is an edge not belonging to $\operatorname{In}\left(P_{j}\right)$. and $\mathbf{x}_{i}$ must be inside the set enclosed by the trajectory from $\mathbf{x}_{j}$ to $\mathbf{x}_{k}$. If $e_{i}$ is completely contained within this set, it cannot be reached 
from $\mathbf{x}_{k}$ without self-intersection of $\xi$. If $e_{i}$ is not fully contained, then it must intersect the trajectory from $\mathbf{x}_{j}$ to $\mathbf{x}_{k}$ and will appear in the signature at some position $m, j<m<k$ and contradict the assumption of abandonment (see figure 8-b). A similar argument holds for the case of a contracting spiral.

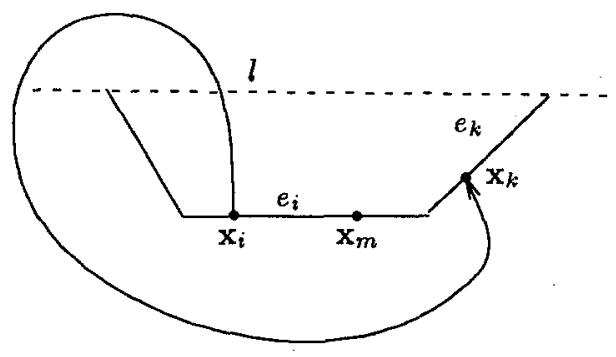

(a)

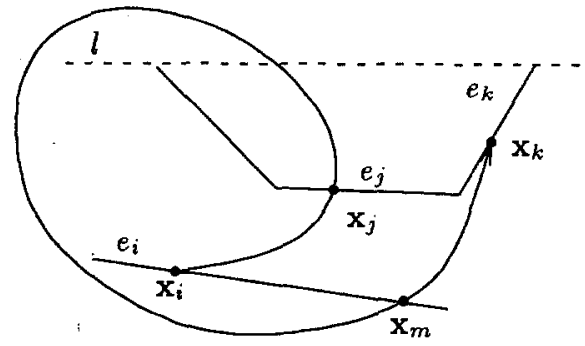

(b)

Fig. 8. Irreversibility of abandonment.

Corollary 5. Every trajectory has an ultimately-periodic signature, i.e., a signature of the form $e_{1}, \ldots, e_{i},\left(e_{i+1}, \ldots, e_{i+j}\right)^{\omega}$ for some finite $i, j$ where $j$ is at most the number of regions.

Proof: Because of the finite number of edges every infinite signature contains a subset $E$ of edges appearing infinitely often. Let $i$ be a position in the signature after all elements of $E$ have already occurred and all the elements of $E(\mathcal{P})-E$ have already disappeared. Let $e=e_{i}$, then the remaining signature can be factorized into $e, \sigma_{1}, e, \sigma_{2}, e \ldots$ where $\sigma_{j} \in(E-\{e\})^{*}$ for every $j>0$. All the elements of $E-\{e\}$ must appear in every $\sigma_{j}$, otherwise they are abandoned. In addition every $\sigma_{j}$ is cycle-free, otherwise $e$ must be abandoned. Finally it is impossible for an edge $e^{\prime}$ to appear before $e^{\prime \prime}$ in $\sigma_{j}$ and after $e^{\prime \prime}$ in $\sigma_{j+1}$ as in the sequence

$$
e \ldots, e^{\prime} \ldots, e^{\prime \prime} \ldots, e \ldots, e^{\prime \prime}, \ldots, e^{\prime}, \ldots, e
$$

because otherwise $e^{\prime}$ will be abandoned due to the cycle $e^{\prime \prime} \ldots, e \ldots, e^{\prime \prime}$. Consequently, all the $\sigma_{j}$ must be identical and the sequence is ultimately-periodic.

Equipped with this nice qualitative property (which does not hold in higher dimensions) we turn to the actual calculation of trajectories of multi-linear systems.

\section{Point-to-point Reachability}

In this section we will devise a framework for representing edges and vertices that will provide for the finite characterization and calculation of the set of points reachable by a trajectory starting at a given point. 
Definition 6 Representations. Let $e$ be an edge with characteristic vectors a and $-\mathbf{a}$. A representation for $e$ consists of two vectors $\mathbf{v} \in \operatorname{cl}(e), \mathbf{u} \in\{\hat{\mathbf{a}},-\hat{\mathbf{a}}\}$, and two numbers $l, h$, such that $l$ is a rational or the special value $-\infty$ and $h>l$ is a rational or $\infty$, and

$$
e=\{\mathbf{v}+\lambda \mathbf{u}: l<\lambda<h\}
$$

The choice of $\mathbf{v}$ and $\mathbf{u}$ implies, of course, the values of $l$ and $h$. Having fixed $\mathbf{v}$ and $\mathbf{u}$ for every edge we can uniquely represent every non-vertex boundary point $\mathbf{x}$ by a pair $(e, \lambda)$ identifying the edge $e$ and the parameter $\lambda$. Every open boundary interval $\left(\mathbf{x}_{1}, \mathbf{x}_{2}\right)$ contained in $e$ is represented by some edge interval $\left(e,\left(\lambda_{1}, \lambda_{2}\right)\right), l \leq \lambda_{1} \leq \lambda_{2} \leq h$. Vertices are represented by themselves.

Definition 7 Successor Function. Let $e$ be and $e^{\prime}$ be two edges with $(\mathbf{u}, \mathbf{v})$ and $\left(\mathbf{u}^{\prime}, \mathbf{v}^{\prime}\right)$-representations. The partial function $f_{e, e^{\prime}}:(l, u) \rightarrow\left(l^{\prime}, u^{\prime}\right)$ is defined as follows: $f_{e, e^{\prime}}(\lambda)=\lambda^{\prime}$ iff $\mathbf{x}^{\prime}=\left(e^{\prime}, \lambda^{\prime}\right)$ is the immediate successor of $\mathbf{x}=(e, \lambda)$.

Claim 3 Given a representation, the successor function is well-defined and computable.

Proof: Obviously if there is no $P$ such that $e \subseteq \operatorname{In}(P)$ and $e^{\prime} \subseteq$ Out $(P)$ then $f_{e, e^{\prime}}$ is nowhere defined. Consider now $(e, \lambda) \in \operatorname{In}(P)$ and its successor $\left(e^{\prime}, \lambda^{\prime}\right) \in$ Out $(P)$ for some region $P$ having a slope c. For $\left(e^{\prime}, \lambda^{\prime}\right)$ to be indeed the successor, the following vector equation must be satisfied for some $t>0$ :

$$
\mathbf{v}^{\prime}+\lambda^{\prime} \mathbf{u}^{\prime}=\mathbf{v}+\lambda \mathbf{u}+t \mathbf{c}
$$

by rearanging, we obtain

$$
\lambda^{\prime} \mathbf{u}^{\prime}=\lambda \mathbf{u}+\left(\mathbf{v}-\mathbf{v}^{\prime}\right)+t \mathbf{c} .
$$

and multiplying both sides by $\hat{c}$, we obtain

$$
\lambda^{\prime}\left(\hat{\mathbf{c}} \cdot \mathbf{u}^{\prime}\right)=\lambda(\hat{\mathbf{c}} \cdot \mathbf{u})+\hat{\mathbf{c}} \cdot\left(\mathbf{v}-\mathbf{v}^{\prime}\right)
$$

from which we get

$$
\lambda^{\prime}=A_{e, e^{\prime}} \lambda+B_{e, e^{\prime}}
$$

where

$$
A_{e, e^{\prime}}=\frac{\mathbf{c} \cdot \mathbf{a}}{\mathbf{c} \cdot \mathbf{a}^{\prime}} \quad \text { and } \quad B_{e, e^{\prime}}=\frac{\hat{\mathbf{c}} \cdot\left(\mathbf{v}-\mathbf{v}^{\prime}\right)}{\mathbf{c} \cdot \mathbf{a}^{\prime}}
$$

By the assumption of nondegeneracy, $\mathbf{c}$ is not parallel to $e^{\prime}$ and therefore the denominator is not zero. If $\lambda^{\prime}$ is outside the interval $\left(l^{\prime}, h^{\prime}\right)$ then the trajectory starting at $(e, \lambda)$ intersect another border element and $f_{e, e^{\prime}}(\lambda)$ is undefined. Similarily we can define a $P$-predecessor function and compute it by

$$
\lambda=\frac{\lambda^{\prime}-B_{e, e^{\prime}}}{A_{e, e^{\prime}}}
$$

The one-step successor function can be generalized naturally to signatures. 
Definition 8 Signature Successor Function. Let $\sigma=e_{1}, \ldots, e_{k}$ be a signature. The signature successor is a partial function $f_{\sigma}:\left(u_{1}, l_{1}\right) \rightarrow\left(u_{k}, l_{k}\right)$ such that $\lambda_{k}=f_{\sigma}\left(\lambda_{1}\right)$ iff a trajectory $\mathbf{x}_{1}, \ldots, \mathbf{x}_{k}$ with $\mathbf{x}_{1}=\left(e_{1}, \lambda_{1}\right)$ has the signature $\sigma$ and $\mathbf{x}_{k}=\left(e_{k}, \lambda_{k}\right)$.

It can be easily verified that $f_{\sigma}$ can be computed recursively from $f_{e_{i}, e_{i+1}}, i=$ $1 \ldots k-1$, yielding

$$
f_{\sigma}\left(\lambda_{1}\right)=A_{\sigma} \lambda_{1}+B_{\sigma}
$$

with

$$
A_{\sigma}=A_{e_{1}, e_{2}} \cdot A_{e_{2}, e_{3}} \ldots A_{e_{k-1}, e_{k}}
$$

and

$$
B_{\sigma}=\left(\cdot\left(\left(B_{e_{1}, e_{2}} \cdot A_{e_{2}, e_{3}}+B_{e_{2}, e_{3}}\right) \cdot A_{e_{3}, e_{4}}+B_{e_{3}, e_{4}}\right) \cdot \cdot\right) \cdot A_{e_{k-1}, e_{k}}+B_{e_{k-1}, e_{k}}
$$

Of particular interest are the successor functions associated with cyclic signatures.

Claim 4 If $\sigma=e_{1}, \ldots, e_{k}$ is an edge cycle, i.e., $e_{k}=e_{1}$, then $A_{\sigma}$ is positive.

Proof: Recalling the classification of edges orientations w.r.t. regions, an edge $e$ is classified as an entry or an exit to $P$ according to the sign of $\mathbf{a} \cdot \mathbf{c}$ where a is the characteristic vector of $e$ relative to $P$. This implies that an edge $e$ can be traversed from $P_{1}$ to $P_{2}$ only if

$$
\frac{\mathbf{c}_{2} \cdot \mathbf{a}}{\mathbf{c}_{1} \cdot \mathbf{a}}>0
$$

where $\mathbf{a}$ is either one of its two characteristic vectors. Now $A_{\sigma}$ is equal to

$$
\frac{\mathbf{c}_{1} \cdot \mathbf{a}_{1}}{\mathbf{c}_{1} \cdot \mathbf{a}_{2}} \cdot \frac{\mathbf{c}_{2} \cdot \mathbf{a}_{2}}{\mathbf{c}_{2} \cdot \mathbf{a}_{3}} \ldots \frac{\mathbf{c}_{n} \cdot \mathbf{a}_{k}}{\mathbf{c}_{k} \cdot \mathbf{a}_{1}}=\frac{\mathbf{c}_{1} \cdot \mathbf{a}_{1}}{\mathbf{c}_{n} \cdot \mathbf{a}_{1}} \cdot \frac{\mathbf{c}_{2} \cdot \mathbf{a}_{2}}{\mathbf{c}_{1} \cdot \mathbf{a}_{2}} \ldots \frac{\mathbf{c}_{k} \cdot \mathbf{a}_{k}}{\mathbf{c}_{k-1} \cdot \mathbf{a}_{k}}
$$

were all the terms are positive.

Suppose $\xi=\mathbf{x}_{1}, \mathbf{x}_{2}, \ldots$ with $\mathbf{x}_{i}=\left(e_{i}, \lambda_{i}\right)$ for every $i=1 \ldots k$, has a periodic signature $\left(e_{1}, \ldots, e_{k-1}\right)^{\omega}$ and let $\sigma=e_{1}, \ldots, e_{k}$. Then the sequence of intersection points of $\xi$ with $e_{1}$ is represented by $\left(e_{1}, \mu_{0}\right),\left(e_{1}, \mu_{1}\right), \ldots$ with $\mu_{0}=\lambda_{1}$ and $\mu_{i+1}=A_{\sigma} \cdot \mu_{i}+B_{\sigma}$. It is straightforward to solve this linear difference equation and obtain the following expression for $\mu_{n}$ :

$$
\mu_{n}= \begin{cases}\mu_{0}+B_{\sigma} \cdot n & \text { if } A_{\sigma}=1 \\ \mu_{0} \cdot A_{\sigma}^{n}+B_{\sigma} \cdot \frac{A_{\sigma}^{n}-1}{A_{\sigma}-1} & \text { otherwise }\end{cases}
$$

We can compute the limit of $\mu_{n}$ as $n$ tends to infinity. This is given by

$$
\mu^{*}= \begin{cases}\mu_{0} & \text { if } A_{\sigma}=1 \text { and } B_{\sigma}=0 \\ \operatorname{sign}\left(B_{\sigma}\right) \cdot \infty & \text { if } A_{\sigma}=1 \text { and }\left|B_{\sigma}\right| \neq 0 \\ \mu_{0} & \text { if } A_{\sigma}>1 \text { and } \mu_{0}=\frac{B_{\sigma}}{1-A_{\sigma}} \\ \operatorname{sign}\left(\mu_{0}+\frac{B_{\sigma}}{A_{\sigma}-1}\right) \cdot \infty & \text { if } A_{\sigma}>1 \text { and } \mu_{0} \neq \frac{B_{\sigma}}{1-A_{\sigma}} \\ \frac{B_{\sigma}}{1-A_{\sigma}} & \text { if } A_{\sigma}<1\end{cases}
$$


For every $e_{i}, i, 1 \leq j<k$ in the signature we define its limit point $\lambda_{i}^{*}$ by letting $\lambda_{1}^{*}=\mu^{*}$ and $\lambda_{i}^{*}=f_{e_{i-1}, e_{i}}\left(\lambda_{i-1}^{*}\right)$. It is clear that the signature $e_{1}, \ldots, e_{k-1}$ can repeat forever only if for every $i, 1 \leq i<k$ all its intersection points with the edge $e_{i}$ are contained in the interval $\left(e_{i},\left(l_{i}, u_{i}\right)\right)$. We can now formulate a criterion for a cycle being repeated infinitely many times.

Criterion 1 (Infinity Test) A trajectory starting with a cycle of the form $\left(e_{1}, \lambda_{1}\right), \ldots,\left(e_{k}, \lambda_{k}\right)$ has a periodic signature $\left(e_{1}, \ldots, e_{k-1}\right)^{\omega}$ iff

$$
l_{i} \leq \lambda_{i}^{*} \leq u_{i} \quad \text { for every } i=1, \ldots, k-1
$$

This criterion is based on the first occurrence of the cycle and enables us to decide in advance whether this cycle will repeat forever or some of its edges will be abandoned after finitely many iterations.

Corollary 9 Point to Point Reachability. It is decidable whether a trajectory starting at $\mathbf{x}=(e, \lambda)$ reaches $\mathbf{x}^{\prime}=\left(e^{\prime}, \lambda^{\prime}\right)$.

Proof: All we need is to calculate the successors and compare them with $\mathbf{x}^{\prime}$. During the generation of the trajectory we keep track of the non-abandoned edges (finitely many). When a cycle is detected the infinity test tells us whether or not this is the terminal cycle. If it is not we continue generating the successors. If it is the terminal cycle we can check whether $e^{\prime}$ is not in the cyclic signature or $\lambda^{\prime}$ is beyond the limit - in that case the answer is negative. Otherwise, after finitely many iterations we either reach $\mathbf{x}^{\prime}$ or bypass it.

Remark: It is not hard to see that this result will hold even if we allow nondeterminism on the boundaries. We will have then a finite number of equivalence classes of trajectories each having the same set of reachable points.

\section{$5 \quad$ Reachability Between Edges}

The results so far allow us to compute the set of all points reachable by a single trajectory. Now we will show how reasoning about a non-countable number of trajectories departing from an edge interval can be reduced to reasoning about a finite number of trajectories.

Claim 5 Let $(e,(l, u))$ be an edge interval. Then if the trajectories starting from $(e, l)$ and $(e, u)$ have identical signatures, so does every trajectory starting at $(e, \lambda)$, for some $l<\lambda<u$.

Proof: Follows from the monotonicity of the successor function and the convexity of the regions.

Now we generalize the notion of successor from single points to intervals.

Definition 10 Edge Successors Set. Let $(e,(l, u)) \subseteq \operatorname{In}(P)$ be an edge interval. The successor set of $e$ is a set of edges and vertices

$$
\left\{\left(e_{1},\left(l_{1}, u_{1}\right)\right), \mathbf{x}_{1},\left(e_{2},\left(l_{2}, u_{2}\right)\right), \mathbf{x}_{2}, \ldots,\left(e_{n},\left(l_{n}, u_{n}\right)\right)\right\}
$$


such that the elements are mutually disjoint, and their union is in one-to-one correspondence with $(e,(l, u))$ via the successor/predecessor functions. (See figure 9).

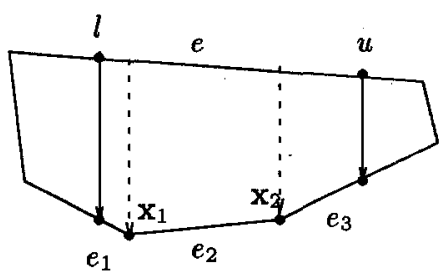

Fig. 9. The successors of $(e,(l, u))$.

Claim 6 The set of edge-successors is finite and computable.

Proof: Because of the computability of the single point successor and predecessor functions. Moreover, for every $i$, all the points in $\left(e_{i},\left(l_{i}, h_{i}\right)\right)$ are the $f_{e, e_{i}}$-images of some sub-interval of $(e,(l, u))$.

Definition 11 Successor Chains. A successor chain is a sequence of edge intervals $\left(e_{1},\left(l_{1}, u_{1}\right)\right),\left(e_{2},\left(l_{2}, u_{2}\right)\right), \ldots$ such that for every $i>1,\left(e_{i+1},\left(l_{i+1}, u_{i+1}\right)\right)$ is an edge-successors of $\left(e_{i},\left(l_{i}, u_{i}\right)\right)$. The set of successor chains starting at an edge interval $\left(e_{1},\left(l_{1}, u_{1}\right)\right)$ can be organized as a successor tree rooted at $\left(e_{1},\left(l_{1}, u_{1}\right)\right)$. Sub-trees have the obvious meaning.

The notion of signature is generalized naturally to successor chains. If a chain has a signature $\sigma$ then there must be at least one trajectory having this signature. This implies that every infinite successor chain must have an ultimately periodic signature.

Claim 7 (Finitely Many Interesting Chains) For every chain in a successor tree, there is some level $i$ such that either the splitting stops (all nodes in levels $j>i$ have exactly one successor) or if they have $s>1$ successors, $s-1$ of these successors already appear at some level $l<i$ in the tree.

Proof: Let $\left(e_{1},\left(l_{1}, u_{1}\right),\left(e_{2},\left(l_{2}, u_{2}\right), \ldots\right.\right.$ be an infinite successor chain with a periodic signature $\sigma^{\omega}, \sigma=\left(e_{1}, \ldots, e_{k-1}\right)$. So $\left(e_{1},\left(l_{1}, u_{1}\right)\right), \ldots,\left(e_{k},\left(l_{k}, u_{k}\right)\right)$ is the first occurrence of this cycle with $e_{1}=e_{k}$ (note that $\left(e_{1},\left(l_{1}, u_{1}\right)\right)$ can have other successors leading to other chains). Let us look at the two sequences $\mu_{0}, \mu_{1} \ldots$ and $\eta_{0}, \eta_{1} \ldots$ defined as $\mu_{0}=l_{k}, \eta_{0}=u_{k}, \mu_{n+1}=f_{\sigma}\left(\mu_{n}\right)$ and $\eta_{n+1}=f_{\sigma}\left(\eta_{n}\right)$. According to our previous analysis there are two principal cases according to the values of $A_{\sigma}, B_{\sigma}, l_{k}$ and $u_{k}$ : 
1. The two sequences have the same limits and thus $\mu_{n}$ satisfies the infinity criterion iff $\eta_{n}$ does. Consequently, if one of them eventually crosses an endpoint of $e$ so does the other and this contradicts the assumption that $\sigma$ is the ultimate period of the signature of the chain. Thus all the intervals $\left(e,\left(\mu_{n}, \eta_{n}\right)\right)$ stay within $e$ and the subtree rooted at $\left(e,\left(l_{k}, u_{k}\right)\right)$ has only one successor chain.

2. The two sequences have different limits - this can only happen when $A_{\sigma}>1$ and $\lambda_{0}=B_{\sigma} /\left(1-A_{\sigma}\right)$ is inside the interval $\left(\mu_{0}, \eta_{0}\right)$ and in this case the sequence $\left(\mu_{n}, \eta_{n}\right)$ of intervals is expanding and $f_{\sigma}\left(\left(\mu_{n}, \lambda_{0}\right)\right)=\left(\mu_{n+1}, \lambda_{0}\right)$ and $f_{\sigma}\left(\left(\lambda_{0}, \eta_{n}\right)\right)=\left(\lambda_{0}, \eta_{n+1}\right)$. Suppose w.l.o.g. that $e$ is bounded from the right by $u$ and unbounded from the left. Then, $\eta_{m}>u$ for some $m>0$ and for every $p>m \eta_{p}=u$ and all the edge intervals will be of the form $\left(e,\left(\mu_{p}, u\right)\right)$ and all of their successors outside $e$ will be, in fact, successors of the same sub-interval $\left(\lambda_{0}, u\right)$ and thus will all be identical.

Claim 8 (Decidability for Periodic Chains) Let $\left(e,\left(l_{1}, u_{1}\right)\right)$ be an edge interval having only one successor chain and let this chain have a periodic signature. Then it is decidable whether there is a trajectory starting from $\left(e,\left(l_{1}, u_{1}\right)\right)$ which intersects another edge interval $\left(e^{\prime},\left(l^{\prime}, u^{\prime}\right)\right)$.

Proof: Obviously if $e^{\prime}$ is not in $\sigma$ then the answer is negative. Otherwise, assume w.l.o.g. that $e^{\prime}=e_{1}$. We calculate the limit $\left(\mu^{*}, \eta^{*}\right)$ of the sequence $\left(\mu_{n}, \eta_{n}\right)$ of intersection intervals with $e_{i}$. If $\left(l^{\prime}, u^{\prime}\right)$ is beyond the limit the answer is negative; otherwise for some finite $i$ either $\left(\mu_{i}, \eta_{i}\right) \cap\left(l^{\prime}, u^{\prime}\right) \neq \emptyset$ and the answer is positive or $\left(\mu_{i}, \eta_{i}\right)$ has "passed" $\left(l^{\prime}, u^{\prime}\right)$.

¿From the last two claims we can deduce:

Theorem 12 Main Result. For every deterministic planar multi-linear system it is decidable whether an edge interval $\left(e^{\prime}\left(l^{\prime}, u^{\prime}\right)\right)$ is reachable from an edge interval $(e,(l, u))$.

Proof: The algorithm develops the successor tree of $(e(l, u))$ where for every edge interval it keeps track of its unabandoned ancestors. Using this information we can detect edge-cycles and calculate their successor functions and limits. When a cycle starting at some $(e,(l, u))$ and having a signature $\sigma$ can repeat forever there are two cases (see claim 7). In the first case we just apply claim 8 directly. In the second case ("expanding" intervals) we develop the successor tree until we reach the end-point(s) of $e$, and from there we can ignore the other branches and consider only the chain with the signature $\sigma^{\omega}$. The algorithm terminates because a terminal non-branching cycle will be encountered along every chain.

As an immediate result we have:

Corollary 13 Reachability is Decidable. The reachability problem between arbitrary finite unions of polygonal sets is decidable.

Proof: Inside the regions we have two-sided determinism so every region-toregion reachability problem can be reduced to a finite number of edge-to-edge reachability problems. 
Acknowledgements We would like to thank A. Bouajjani and J. Sifakis for many helpful comments.

\section{References}

1. R. Alur, C. Courcoubetis, and D.L. Dill. Model checking for real-time systems. In Proceedings of the Fifth Annual Symposium on Logic in Computer Science, pages 414-425. IEEE Computer Society Press, 1990.

2. R. Alur, G. Courcoubetis, T. Henzinger, and P. Ho, Hybrid automata: An algorithmic approach to the specification and analysis of hybrid systems. In A. Ravn and H. Rischel, editors, Workshop on Hybrid Systems, Lect. Notes in Comp. Sci. Springer-Verlag, 1993.

3. T. Henzinger, X. Nicollin, J. Sifakis, and S. Yovine. Symbolic model-checking for real-time systems. In Proceedings of LICS'92, 1992.

4. Y. Kesten, A. Pnueli, J. Sifakis, and S. Yovine. Integration graphs: A class of decidable hybrid systems. In A. Ravn and H. Rischel, editors, Workshop on Hybrid Systems, Lect. Notes in Comp. Sci. Springer-Verlag, 1993.

5. O. Maler, Z. Manna, and A. Pnueli. From timed to hybrid systems. In J.W. de Bakker, C. Huizing, W.P. de Roever, and G. Rozenberg, editors, Proceedings of the REX Workshop "Real-Time: Theory in Practice", volume 600 of Lect. Notes in Comp. Sci., pages 447-484. Springer-Verlag, 1992.

6. Z. Manna and A. Pnueli. Verifying hybrid systems. In A. Ravn and H. Rischel, editors, Workshop on Hybrid Systems, Lect. Notes in Comp. Sci. Springer-Verlag, 1993.

7. X. Nicollin, A. Olivero, J. Sifakis, and S. Yovine. An approach to the description and analysis of hybrid systems. In A. Ravn and H. Rischel, editors, Workshop on Hybrid Systems, Lect. Notes in Comp. Sci. Springer-Verlag, 1993.

8. X. Nicollin, J. Sifakis, and S. Yovine. From ATP to timed graphs and hybrid systems. In J.W. de Bakker, C. Huizing, W.P. de Roever, and G. Rozenberg, editors, Proceedings of the REX Workshop "Real-Time: Theory in Practice", volume 600 of Lect. Notes in Comp. Sci., pages 549-572. Springer-Verlag, 1992. 